\title{
Transport of groundwater contaminants with multiple reactive species
}

\author{
Nirmala P. Ratchagar* and S. Senthamilselvi** \\ *Department of Mathematics, Annamalai University, Annamalainagar, India \\ **Department of Mathematics, SRASC, Chidambaram, India \\ nirmalapasala@yahoo.co.in,msselvi2305@yahoo.com
}

\begin{abstract}
The motive of this work is to give a mathematical model describing contaminant transport problem for three reacting species coupled by a sequential first order reaction. The governing equations are transformed into a nondimensional form using suitable set of dimensionless variables and solved by perturbation method. The effect of various physical parameters involved in the problem are presented graphically to illustrate interesting features of the solutions.
\end{abstract}

Key words: Multispecies, Reactive transport, First-order reaction.

\section{Introduction}

The groundwater acts as a major source of drinking water. The demand of groundwater supply is increasing day by day into various areas such as domestic needs, industries, agriculture and so on. Accidental effluence is one of the major sources of hazardous water and often leads to groundwater contamination. The ever increasing industrialization, urbanization, excessive use of agriculture fertilizers and water dumping activities makes the environment and groundwater polluted. Groundwater resources have become increasingly threatened by the leaching of contaminants from uncontrolled landfills containing industrial and/or household waste, infiltration of pesticides and fertilizers from agricultural areas and leakage of a wide range of organic pollutants from petrol stations, refineries and pipelines $[1,2,3]$.

Hazardous waste consist of various organic and inorganic contaminants and generally found near industrial sites. Contaminants persist into the groundwater more than desired limits are poisonous and affect the human as well as animal health. Therefore it is essential to predict the behaviour of chemical contaminants in flowing groundwater. Modeling the contaminant transport for multiple reactive contaminants in groundwater aquifers has received considerable attention in recent years. Further this modeling is inevitable for the comprehensive understanding of the processes occurring in groundwater. In 
most instances, sequential first order reaction models have been used to describe reactive transport in many groundwater contamination problems. For example, chlorinated solvent contaminants which include multiple parent and daughter species, are common reactive contaminants observed at several hazardous waste sites. At these sites use of single-species model to analyze the fate and transport mechanism of a (parent) species, is not adequate to evaluate the overall impacts. To accomplish such description, a mathematical model predicting the simultaneous reactive transport of microbes, substrates, and contaminants within groundwater is required.

Microbial analysis of groundwater samples collected from different field sites show elevated levels of bacteria in the samples from biologically active contaminated aquifers as compared to Pristine aquifers[4]. The rate at which contaminants are used within the bio-reactive zone is a function of bacterial concentration. This concentration is in turn a function of bacterial growth and transport processes $[5,6]$.

Recently the transport of common environmental contaminants, such as chlorinated solvents[7] and metals, have established a need for a more general purpose of reactive transport which is capable of modeling multispecies reactive transport of different types of contaminants. Multi-species, bioreactive transport in one-dimensional soil columns has been modeled by several researchers $[8,9,10,11]$. Rifai et al.[12] developed a two-species bioremediation model, known as BIOPLUME-II, to predict the fate and transport of hydrocarbon contaminants that degrade via aerobic reactions. Jennifer et al.[13] presented a reactive transport model for geochemical mitigation of CO2 leaking into a confined aquifer.

The objective of the present work is to develop the analytical solution for a multi-species advection-dispersion transport equation comprising of sequential first-order reactions. In this work the daughter products are assumed to be produced by a single parent species. Perturbation technique has been adopted for solving the equations. The porous parameter, Schmidt number and Damkohler number are all considered so as to extensively investigate their distinct influence on the velocity and concentration fields. 


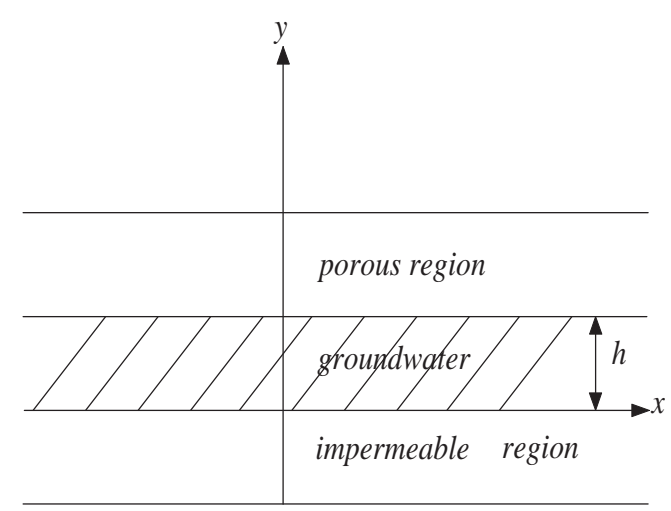

Figure 1: Physical Configuration

\section{Problem Formulation}

We consider transient laminar flow of a viscous, incompressible fluid (groundwater) of uniform cross-section h, lying below the porous layer and above the impermeable layer. The groundwater is assumed to be contaminated. The schematic diagram of the problem is shown in figure 1 .

The governing equations of continuity and momentum are:

$$
\begin{aligned}
& \frac{\partial u}{\partial x}+\frac{\partial v}{\partial y}=0 \\
& \frac{\partial u}{\partial t}+u \frac{\partial u}{\partial x}=-\frac{1}{\rho} \frac{\partial p}{\partial x}+\nu\left(\frac{\partial^{2} u}{\partial x^{2}}+\frac{\partial^{2} u}{\partial y^{2}}\right)-\frac{\nu}{k} u
\end{aligned}
$$

where,

$u$ is the velocity of the fluid particle, $\rho$ is the density of the fluid, $p$ is the pressure, $t$ is time, $\nu$ is the kinematic viscosity of fluid, $k$ is the permeability of the porous medium.

The initial and boundary conditions are given by:

$$
\begin{gathered}
u=0 \text { at } t=0, \forall y \\
u=u_{0}\left(1+\epsilon e^{\mathrm{i}(\lambda x+\omega t)}\right) \text { at } y=0, t>0 \\
\frac{\partial u}{\partial y}=\frac{\alpha}{\sqrt{k}} u \text { at } y=h, t>0
\end{gathered}
$$


where,

$u_{0}$ is the initial velocity,

$\epsilon$ is the perturbation parameter,

$\lambda$ is the stream-wise wave number,

$\omega$ is the frequency parameter,

i represents the imaginary part,

$\alpha$ is slip parameter(dimensionless quantity).

Non-dimensionalizing the above equations (1) and (2) together with the initial and boundary conditions (3) to (5) using the variables,

$x^{*}=\frac{x}{h}, y^{*}=\frac{y}{h}, t^{*}=\frac{t \nu}{h^{2}}, u^{*}=\frac{h u}{\nu}, v^{*}=\frac{h v}{\nu}, p^{*}=\frac{p}{\rho\left(\frac{\nu}{h}\right)^{2}}, \omega^{*}=\frac{\omega h^{2}}{\nu}, \lambda^{*}=\lambda h, u_{0}^{*}=\frac{h u_{0}}{\nu}$

we get,

$\frac{\partial u}{\partial x}+\frac{\partial v}{\partial y}=0$

$\frac{\partial u}{\partial t}+u \frac{\partial u}{\partial x}=-\frac{\partial p}{\partial x}+\left(\frac{\partial^{2} u}{\partial x^{2}}+\frac{\partial^{2} u}{\partial y^{2}}\right)-\sigma^{2} u$

where $\sigma^{2}=\frac{h^{2}}{k}$, porous parameter.

The boundary condition becomes,

$$
\begin{gathered}
u=0 \quad \text { at } t=0, \forall y \\
u=u_{b}\left(1+\epsilon e^{\mathbf{i}(\lambda x+\omega t)}\right) \text { at } y=0 \\
\frac{\partial u}{\partial y}=\alpha \sigma u \text { at } y=1
\end{gathered}
$$

Equation(7) together with the boundary conditions (8) and (9) are solved using the perturbation technique.

The movement of the three species takes place in the infinite region $0<x<\infty$ with a first-order chemical reaction. In general, it is assumed that each species (other than the original species) is produced by a first-order reaction with the subsequent species. For example, species $i+1$ which is produced by first-order reaction of species $\mathrm{i}$, also reacts linearly to produce species $i+2$ which in turn, reacts to produce species $i+3$ and so forth. In this paper we have taken for $i=3$. For a sequential reactive transport of a three species system, the governing transport equations are described by: 


$$
\begin{gathered}
\frac{\partial c_{1}}{\partial t}+u \frac{\partial c_{1}}{\partial x}=D\left(\frac{\partial^{2} c_{1}}{\partial x^{2}}+\frac{\partial^{2} c_{1}}{\partial y^{2}}\right)-k_{1} c_{1} \\
\frac{\partial c_{2}}{\partial t}+u \frac{\partial c_{2}}{\partial x}=D\left(\frac{\partial^{2} c_{2}}{\partial x^{2}}+\frac{\partial^{2} c_{2}}{\partial y^{2}}\right)-k_{2} c_{2}+y_{1} k_{1} c_{1} \\
\frac{\partial c_{3}}{\partial t}+u \frac{\partial c_{3}}{\partial x}=D\left(\frac{\partial^{2} c_{3}}{\partial x^{2}}+\frac{\partial^{2} c_{3}}{\partial y^{2}}\right)-k_{3} c_{3}+y_{2} k_{2} c_{2}
\end{gathered}
$$

where,

$c_{1}, c_{2}, c_{3}$ are concentrations of the first, second and third species respectively,

$k_{1}, k_{2}, k_{3}$ are first order reaction rates,

$y_{1}, y_{2}$ are the stoichiometric yield coefficients,

$D$ is the diffusion coefficient,

The first term on the left hand side of each of equations (10)-(12) represents the transient or accumulation effect. The second term on the left hand side of each of these equations represent the advection effect which is defined as the transport of contaminant species by the velocity. The first term on the right hand side of the equations accounts for the dispersion or diffusion effect which is responsible for the spreading of the contaminant species in the medium. The last term of equation (10) represents the first-order reaction effect which takes place in the domain. The last two terms of equations (11) and (12) represent the first-order sequential reaction chain effect which are of the same order.

The initial and boundary conditions for this problem are :

$$
\begin{aligned}
& \frac{\partial c_{1}}{\partial y}=0, \quad \frac{\partial c_{2}}{\partial y}=0, \quad \frac{\partial c_{3}}{\partial y}=0 \quad \text { at } y=0, t>0 \\
& \left.\begin{array}{l}
c_{1}=c_{b_{1}}\left(1+\epsilon e^{\mathbf{i}(\lambda x+\omega t)}\right) \\
c_{2}=c_{b_{2}}\left(1+\epsilon e^{\mathbf{i}(\lambda x+\omega t)}\right) \\
c_{3}=c_{b_{3}}\left(1+\epsilon e^{\mathbf{i}(\lambda x+\omega t)}\right)
\end{array}\right\} \quad y=h, t>0
\end{aligned}
$$

where, $c_{b_{1}}, c_{b_{2}}, c_{b_{3}}$ are initial concentrations.

It is convenient to work with dimensionless equations. This can be accomplished by using 
$C_{i}^{*}=\frac{c_{i}}{c_{0}}, \quad u^{*}=\frac{h u}{\nu}, \quad v^{*}=\frac{h v}{\nu}, \quad y^{*}=\frac{y}{h}, \quad x^{*}=\frac{x}{h}, \quad t^{*}=\frac{t \nu}{h^{2}}, \quad C_{b_{i}}^{*}=\frac{c_{b_{i}}}{c_{0}}$

where, $i=1,2,3$ and $h, c_{0}$ are characteristic distance and concentration respectively.

Substituting equation (15) into equations (10)-(14) gives, respectively:

$\frac{\partial C_{1}}{\partial t}+u \frac{\partial C_{1}}{\partial x}=\frac{1}{S c}\left(\frac{\partial^{2} C_{1}}{\partial x^{2}}+\frac{\partial^{2} C_{1}}{\partial y^{2}}\right)-D a_{1} C_{1}$

$\frac{\partial C_{2}}{\partial t}+u \frac{\partial C_{2}}{\partial x}=\frac{1}{S c}\left(\frac{\partial^{2} C_{2}}{\partial x^{2}}+\frac{\partial^{2} C_{2}}{\partial y^{2}}\right)-D a_{2} C_{2}+y_{1} D a_{1} C_{1}$

$\frac{\partial C_{3}}{\partial t}+u \frac{\partial C_{3}}{\partial x}=\frac{1}{S c}\left(\frac{\partial^{2} C_{3}}{\partial x^{2}}+\frac{\partial^{2} C_{3}}{\partial y^{2}}\right)-D a_{3} C_{3}+y_{2} D a_{2} C_{2}$

Initial condition: $\quad C_{1}=0, \quad C_{2}=0, \quad C_{3}=0 \quad$ at $\quad t=0$

Boundary conditions:

$$
\left.\begin{array}{rl}
\frac{\partial C_{1}}{\partial y}=0, & \frac{\partial C_{2}}{\partial y}=0, \frac{\partial C_{3}}{\partial y}=0 \text { at } y=0 \\
& C_{1}=C_{b_{1}}\left(1+\epsilon e^{\mathrm{i}(\lambda x+\omega t)}\right) \\
& C_{2}=C_{b_{2}}\left(1+\epsilon e^{\mathrm{i}(\lambda x+\omega t)}\right) \\
& C_{3}=C_{b_{3}}\left(1+\epsilon e^{\mathrm{i}(\lambda x+\omega t)}\right)
\end{array}\right\} \quad \mathrm{y}=1, t>0
$$

where, $D a_{1}=\frac{k_{1} h^{2}}{\nu}, \quad D a_{2}=\frac{k_{2} h^{2}}{\nu}, \quad D a_{3}=\frac{k_{3} h^{2}}{\nu}, \quad S c=\frac{\nu}{D}$ are Damkohler numbers for the first, second and third species and Schmidt number respectively. 


\section{Solution Method}

We use here the perturbation method to derive solutions of coupled multiple species transport equations. To accomplish this method we define a small geometric parameter $(\epsilon<<1)$ as :

$$
\begin{gathered}
u(x, y, t)=u_{0_{i}}(y)+\epsilon e^{\mathbf{i}(\lambda x+\omega t)} u_{1}(y)+o\left(\epsilon^{2}\right) \\
p(x, y, t)=p_{0}(x)+\epsilon e^{\mathbf{i}(\lambda x+\omega t)} p_{1}(y)+o\left(\epsilon^{2}\right) \\
C_{i}(x, y, t)=C_{0_{i}}(y)+\epsilon e^{\mathbf{i}(\lambda x+\omega t)} C_{1_{i}}(y)+o\left(\epsilon^{2}\right)
\end{gathered}
$$

where, $i=1,2,3$ represents the concentration of first, second and third species respectively. Substituting equations (21)-(23) into equations (7), (16)-(18) and collecting the coefficient of $\epsilon^{0}$ and $\epsilon$ on both sides, we obtain the following set of equations:

\section{Base state equations:}

$\frac{d^{2} u_{0}}{d y^{2}}-\sigma^{2} u_{0}=g_{1}$

$\frac{1}{S c} \frac{d^{2} C_{0_{1}}}{d y^{2}}-D a C_{0_{1}}=0$

$\frac{1}{S c} \frac{d^{2} C_{0_{2}}}{d y^{2}}-D a C_{0_{2}}+y_{1} D a C_{0_{1}}=0$

$\frac{1}{S c} \frac{d^{2} C_{0_{3}}}{d y^{2}}-D a C_{0_{3}}+y_{2} D a C_{0_{2}}=0$

subject to the boundary conditions :

$$
\begin{gathered}
u_{0}=u_{b} \text { at } y=0 \\
\frac{d u_{0}}{d y}=\alpha \sigma u_{0} \text { at } y=1 \\
\frac{d C_{0_{1}}}{d y}=0, \frac{d C_{0_{2}}}{d y}=0, \frac{d C_{0_{3}}}{d y}=0 \text { at } y=0 \\
C_{0_{1}}=C_{b_{1}}, C_{0_{2}}=C_{b_{2}}, C_{0_{3}}=C_{b_{3}} \text { at } y=1
\end{gathered}
$$

where, $u_{b}, C_{b_{1}}, C_{b_{2}}, C_{b_{3}}$ are constants.

Assuming that the relative reaction rates are affected by the stoichiometry of the reaction scheme we use one Damkohler number i.e. $D a_{1}=D a_{2}=D a_{3}=D a[14]$. 
The solutions to the base state equations of velocity and concentration of three species reactive transport are given by

$$
\begin{aligned}
u_{0} & =g_{2} e^{\sigma y}+g_{3} e^{-\sigma y}-\frac{g_{1}}{\sigma^{2}} \\
C_{0_{1}} & =A e^{\sqrt{D a S c} y}+B e^{-\sqrt{D a S c} y} \\
C_{0_{2}} & =A_{1} e^{\sqrt{D a S c} y}+B_{1} e^{-\sqrt{D a s c} y}-\frac{A y_{1} D a y \cosh \sqrt{D a S c} y}{\sqrt{D a S c}} \\
C_{0_{3}} & =A_{3} e^{\sqrt{D a S c} y}+B_{3} e^{-\sqrt{D a S c} y}-\frac{y_{2} D a S c A_{1} y e^{\sqrt{D a S c}} y}{2 \sqrt{D a S c}}+\frac{y_{2} D a S c B_{1} y e^{-\sqrt{D a S c}} y}{2 \sqrt{D a S c}} \\
& +\frac{1}{4 D a S c} A y_{1} y_{2} D a^{2} S c e^{\sqrt{D a S c} y}\left(\frac{y^{2}}{2}-\frac{y}{2 \sqrt{D a S c}}\right) \\
& -\frac{1}{4 D a s c} A y_{1} y_{2} D a^{2} S c e^{-\sqrt{D a S c}} y\left(\frac{y^{2}}{2}+\frac{y}{2 \sqrt{D a S c}}\right)
\end{aligned}
$$

where, $g_{1}=\frac{\partial p_{0}}{\partial x}, \quad g_{2}=-\frac{1}{e^{\sigma(\alpha-1)}}\left(g_{3} e^{-\sigma}(\alpha+1)+\frac{\alpha g_{1}}{\sigma^{2}}\right)$,

$$
\begin{aligned}
g_{3} & =u_{b}+\frac{g_{3} e^{-\sigma}(\alpha+1)}{e^{\sigma}(\alpha-1)}+\frac{g_{1}}{\sigma^{2}}\left(\frac{\alpha}{e^{\sigma}(\alpha-1)+1}\right), \\
A & =B=\frac{C_{b_{1}}}{2 \cosh \sqrt{D a S c}}, \\
A_{1} & =B_{1}+\frac{A y_{1} D a}{D a S c} \\
B_{1} & =\frac{1}{2 \cos h \sqrt{D a S c}}\left(C_{b_{2}}-\frac{A y_{1} D a}{D a s c} e^{\sqrt{D a S c}}-\frac{A y_{1} D a \cosh \sqrt{D a S c}}{\sqrt{D a s c}}\right), \\
A_{3} & =B_{3}, \\
B_{3} & =\frac{1}{2 \cosh \sqrt{D a S c}}\left(\frac{y_{2} D a S c}{2 \sqrt{D a S c}}\left(A_{1} e^{\sqrt{D a S c}}-B_{1} e^{-\sqrt{D a S c}}\right)\right. \\
& -\frac{1}{4 D a S c} A y_{1} y_{2} D a^{2} S c e^{\sqrt{D a S c}}\left(\frac{1}{2}-\frac{1}{2 \sqrt{D a S c}}\right) \\
& \left.+\frac{1}{4 D a S c} A y_{1} y_{2} D a^{2} S c e^{-\sqrt{D a S c}}\left(\frac{1}{2}+\frac{1}{2 \sqrt{D a S c}}\right)+C_{b_{3}}\right)
\end{aligned}
$$

Collecting the coefficient of $\epsilon^{1}$ gives the perturbed part.

\section{First order equations:}

$$
\begin{aligned}
& \frac{\partial^{2} u_{1}}{\partial y^{2}}+\left(\omega \tan (\lambda x+\omega t)-\lambda^{2}\right) u_{1}+\left(\lambda \tan (\lambda x+\omega t)-\sigma^{2}\right) u_{0} u_{1}+\lambda p_{1} \tan (\lambda x+\omega t)=0 \\
& \frac{1}{S c} \frac{d^{2} C_{1_{1}}}{d y^{2}}+\left(\omega \tan (\lambda x+\omega t)+\lambda \tan (\lambda x+\omega t) u_{0}+\frac{1}{S c} \lambda^{2}+D a\right) C_{1_{1}}=0
\end{aligned}
$$




$$
\begin{aligned}
& \overline{S c} \frac{d^{2} C_{1_{2}}}{d y^{2}}+\left(\omega \tan (\lambda x+\omega t)+\lambda \tan (\lambda x+\omega t) u_{0}-\frac{1}{S c} \lambda^{2}-D a\right) C_{1_{2}}+y_{1} D a C_{1_{1}}=0 \\
& \frac{1}{S c} \frac{d^{2} C_{1_{3}}}{d y^{2}}+\left(\omega \tan (\lambda x+\omega t)+\lambda \tan (\lambda x+\omega t) u_{0}-\frac{1}{S c} \lambda^{2}-D a\right) C_{1_{3}}+y_{2} D a C_{1_{2}}=0
\end{aligned}
$$

subject to the boundary conditions,

$$
\begin{aligned}
& u_{1}=u_{b} \text { at } y=0, \\
& \frac{d u_{1}}{d y}=\alpha \sigma u_{1} \text { at } y=1 \\
& \frac{d C_{1_{1}}}{d y}=0, \frac{d C_{1_{2}}}{d y}=0, \frac{d C_{1_{3}}}{d y}=0 \text { at } y=0 \\
& C_{1_{1}}=C_{b_{1}}, \quad C_{1_{2}}=C_{b_{2}}, \quad C_{1_{3}}=C_{b_{3}} \text { at } y=1
\end{aligned}
$$

The above equations with the corresponding boundary conditions, are solved numerically. On solving these equations we get $u_{1}, C_{1_{1}}, C_{1_{2}}$ and $C_{1_{3}}$. Considering the real part and neglecting the higher order terms (order of $\epsilon^{2}$ ) we get the solution. The sum of the base part and perturbed part gives the required velocity and concentration of species 1,2 and 3 respectively as:

$$
\begin{aligned}
& u_{1}=u_{0}+\epsilon \cos (\lambda x+\omega t) u_{1} \\
& C_{1}=C_{0_{1}}+\epsilon \cos (\lambda x+\omega t) C_{1_{1}} \\
& C_{2}=C_{0_{2}}+\epsilon \cos (\lambda x+\omega t) C_{1_{2}} \\
& C_{3}=C_{0_{3}}+\epsilon \cos (\lambda x+\omega t) C_{1_{3}}
\end{aligned}
$$

\section{Discussion of Results:}

In this section, a representative set of numerical results are presented in figures 2 to 5 . These results are chosen to illustrate the effects of the porous parameter $\sigma$, Damkohler number $D a$ and Schmidt number $S c$.

Figure 2 shows the effect of porous parameter $\sigma$ on velocity. From this figure it is observed that the velocity detends with increase in porous parameter. This result is explained by the fact that the presence of a porous medium increases the resistance to fluid flow and thus the velocity is decreasing. 
Figure 3 presents typical transient state concentration profiles for the three species considered in the study at various values of the Schmidt number Sc. Schmidt number is a dimensionless number defined as the ratio of momentum diffusivity and mass diffusivity. As Sc increases, for the multiple reactive flow, values are strongly reduced, since larger values of Sc are equivalent to a reduction in the chemical molecular diffusivity (i.e) less diffusion therefore takes place by mass transport. It is predicted that increase in the Schmidt number decrease the concentration level of all species. This is due to the movement of the plume-like concentration profiles in the direction of flow and is accompanied by decrease in the concentration boundary layer thickness of all species. The physical meaning of this is multi-species transport with coupled first-order chemical reaction reaches the transient-state conditions at a faster rate.

The three species concentration profile for different values of Damkohler number is displayed through figure 4. Increasing values of chemical reaction cause decrease in the concentration level of parent species and daughter species. The reason behind this is the relative concentration of the second and third species is low since the domain is dispersion dominant due to high dispersion coefficient. This is due to the fact that the substantial increase in the rate of solute transfer in presence of chemical reaction which enhances the mass transfer.

Figure 5 depict the concentration profile for the reactive species at various heights. It is seen that increase in the chemical reaction order intends the concentration level of all species with respect to height. This is due to the movement of the concentration profiles in the axial distance as the reaction order increases. As the chemical reaction order increases, the concentration levels of all species increases over most of the transport region. However, it is predicted that, in general, increasing-type scale-dependent dispersion and transport velocity have significant reduction effects on the concentration level of all species.

\section{Conclusion :}

In this paper, the study is focused on the modeling of a three-species sequential reactive contaminant transport with first order reaction rate, and the sequential coupled reactions are assumed linear. The present model is a useful tool for analyzing different types of subsurface contaminant reactions, microbial metabolisms and microbial transport kinetics that aid in natural attenuation 
of contaminants. Predictions from the model are used for screening remediation alternatives and for forecasting contaminant exposure levels and environmental risks.

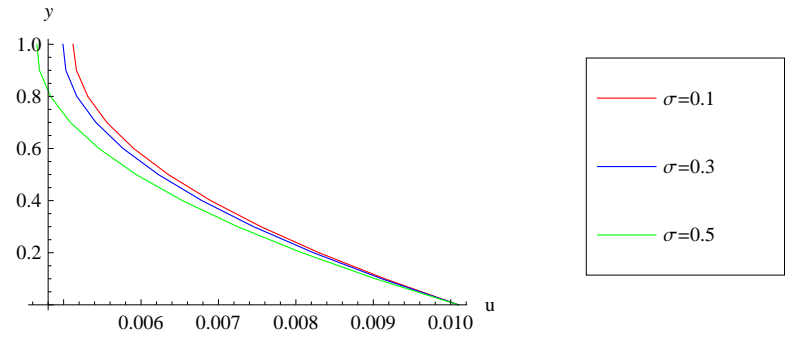

Figure 2: Velocity for different porous parameter

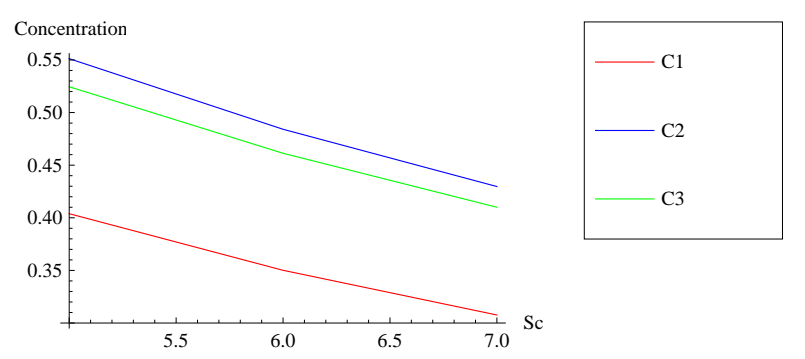

Figure 3: Concentration for fixed height of different Schmidt number

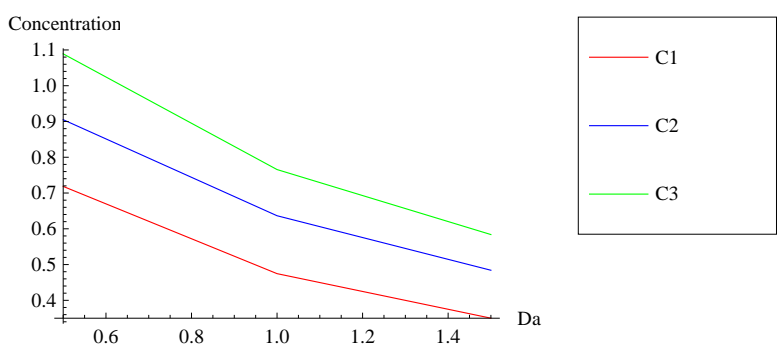

Figure 4: Concentration for fixed height of different Damkohler number

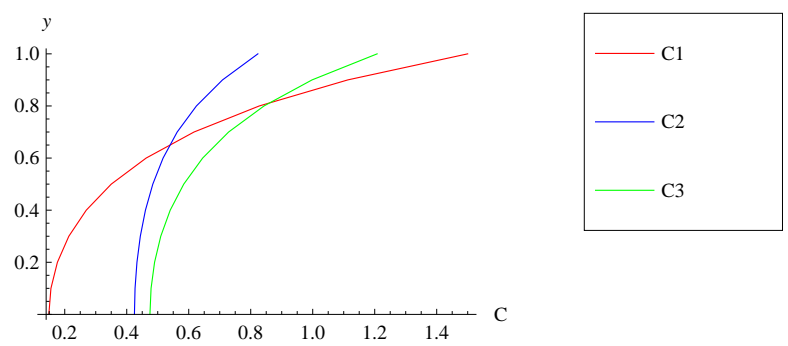

Figure 5: Concentration distribution for the reactive species at different heights 


\section{References}

[1] Trauth, R and Xanthopoulos, C. Non-point pollution of groundwater in urban areas, Water Research, 31(11), 2711-2718, 1997.

[2] Anderson, P., Cunningham, C.J., Barry, D.A., Gravimetric analysis of organic contamination in railway ballast, Land Contamination and Reclamation 8(2), 71-74, 2000.

[3] Babiker, I.S., Mohamed, A.A.M., Terao, H., Kato, K., Ohta, K., Assessment of groundwater contamination by nitrate leaching from intensive vegetable cultivation using geographical information system, Environment International 29 (8), 1009-1017, 2004.

[4] Hemmingsen, B.B and Randall, J.D. Bacterial population changes associated with bioremediation of hydrocarbon contaminated soil and water, Emerging Technologies in Hazardous waste management VIII,, ACS ISEC Special Symposium, 46-49, 1996.

[5] Taylor, S.W., and Jaffe, P. R. Biofilm growth and the related changes in the physical properties of a porous medium ,, Experimental investigations, Water resources Research 26, no.9:2153-2159, 1990.

[6] Reddy, H.L., and Ford,R.M. Analysis of biodegradation and bacterial transport: Comparison of Models with kinetic and equilibrium bacterial adsorbtion, Journal of contaminant Hydrology, 22, 271-287, 1996.

[7] Semprini, L.,Kitnadis,R.M., Kampbell,D. and Wilson,J. Anaerobic Transformation of Chlorinated aliphatic hydorcarbons in a sand aquifer based on spatial chemical distribution., Water Resources Research 31, no.4:1051-1062, 1995.

[8] Molz,F.J., Winddowson,M.A. and Benefield,L.D. Simulation of microbial growth dynamics coupled to nutrient oxygen transport in porous media., Water Resources Research 22, no. 8:1207-1216, 1986.

[9] Zysset,A., Stauffer,F. and Dracos,T.Modeling of reactive groundwater transport governed by biodegradation., Water Resources Research 30, no. 8:1207-1216, 1994.

[10] Clement,T.P.,Hooker,B.S.and Skeen,R.S.Numerical Modeling of biologically reactive transport near a nutrient injection well., ASCE Journal Environment Engineering Division 122, no.9:833-839, 1996a. 
[11] Clement, T.P., Peyton,B.M., Skeen,R.S.,Jennings,D.A. and Petersen,J.N. Microbial growth and transport in porous media under dentrification conditions:Experiment and Simulations Journal of Contaminant Hydrology 24, 269-285, 1997.

[12] Rifai, S.H., Bedient, P.B., Borden, R.C., Haasbeek, J.F., BIOPLUME II Computer model of two-dimensional contaminant transport under the influence of oxygen limited biodegradation in ground water, National Center for Ground Water Research, Rice University, 1987.

[13] Jennifer L. Druhan, Stphanie Viallea, Kate Mahera, Sally Bensonb $A$ reactive transport model for geochemical mitigation of CO2 leaking into a confined aquifer, Energy Procedia 63, 4620-4629, 2014.

[14] Shah, S.I.A., Kostiuk, L.W. and Kresta,S.M., The effects of mixing, reaction rates and stoichiometry on yield for mixing sensitive reactions Part I:Model Development, International journal of chemical engineering, 1-17, 2012. 